\title{
The regulatory effect of miRNAs is a heritable genetic trait in humans
}

\author{
Paul Geeleher ${ }^{1}$, R Stephanie Huang ${ }^{2}$, Eric R Gamazon ${ }^{3}$, Aaron Golden ${ }^{4}$ and Cathal Seoighe ${ }^{1,5^{*}}$
}

\begin{abstract}
Background: microRNAs (miRNAs) have been shown to regulate the expression of a large number of genes and play key roles in many biological processes. Several previous studies have quantified the inhibitory effect of a miRNA indirectly by considering the expression levels of genes that are predicted to be targeted by the miRNA and this approach has been shown to be robust to the choice of prediction algorithm. Given a gene expression dataset, Cheng et al. defined the regulatory effect score (RE-score) of a miRNA as the difference in the gene expression rank of targets of the miRNA compared to non-targeted genes.
\end{abstract}

Results: Using microarray data from parent-offspring trios from the International HapMap project, we show that the RE-score of most miRNAs is correlated between parents and offspring and, thus, inter-individual variation in RE-score has a genetic component in humans. Indeed, the mean RE-score across miRNAs is correlated between parents and offspring, suggesting genetic differences in the overall efficiency of the miRNA biogenesis pathway between individuals. To explore the genetics of this quantitative trait further, we carried out a genome-wide association study of the mean RE-score separately in two HapMap populations (CEU and YRI). No genome-wide significant associations were discovered; however, a SNP rs 17409624, in an intron of DROSHA, was significantly associated with mean RE-score in the CEU population following permutation-based control for multiple testing based on all SNPs mapped to the canonical miRNA biogenesis pathway; of 244 individual miRNA RE-scores assessed in the CEU, 214 were associated $(p<0.05)$ with rs 17409624. The SNP was also nominally significantly associated $(p=0.04)$ with mean RE-score in the YRI population. Interestingly, the same SNP was associated with 17 (8.5\% of all expressed) miRNA expression levels in the CEU. We also show here that the expression of the targets of most miRNAs is more highly correlated with global changes in miRNA regulatory effect than with the expression of the miRNA itself.

Conclusions: We present evidence that miRNA regulatory effect is a heritable trait in humans and that a polymorphism of the DROSHA gene contributes to the observed inter-individual differences.

\section{Background}

microRNAs (miRNAs) are a class of small non-coding RNA molecules of approximately 21 nucleotides in length that regulate gene expression. They typically bind to complementary loci in the $3^{\prime}$ untranslated region (UTR) of mRNA and prevent translation to mature protein. An individual miRNA can regulate the expression of hundreds of genes. Some genes, particularly those with longer $3^{\prime}$ UTRs, are often the targets of multiple miRNAs and

\footnotetext{
*Correspondence: cathal.seoighe@nuigalway.ie

${ }^{1}$ Department of Mathematics, Statistics and Applied Mathematics, National

University of Ireland, Galway, Ireland

5 Institute of Infectious Disease and Molecular Medicine, University of Cape

Town, Cape Town 7925, South Africa

Full list of author information is available at the end of the article
}

consequently, miRNA mediated regulation tends to result in the fine tuning of the expression of many proteins within a cell $[1,2]$. In mammals, miRNAs are thought to regulate the expression of as many as $50 \%$ of protein coding genes [3]. miRNA expression impacts on almost every cellular process and miRNA dysregulation has been implicated in many pathologies $[1,4]$.

miRNAs regulate a range of biological pathways associated with cancer including apoptosis [5] and cell proliferation [6]; dysregulation of miRNAs has also been widely observed in cancer [7]. For example over expression of miR-155 has been implicated in Hodgkin's and Burkitt's lymphoma [8], while miR-15 and miR-16, which target the anti-apoptotic gene BCL2, have been shown to be dysregulated in chronic lymphocytic leukemia [9]. miRNAs 
have been found in many of the genomic regions associated with chromosomal abnormalities in cancer, including regions of amplification, which may contain oncogenes, regions of loss of heterozygosity, which may harbor tumor suppressor genes and fragile sites which are preferential sites for translocation, deletion, amplification, sister chromatid exchange and insertion of tumor associated viruses like human papilloma virus [10].

While many specific maturation steps have been uncovered for different miRNAs, most known human miRNAs are processed in the same way by the miRNA biogenesis pathway. miRNA precursors, known as primary miRNA (pri-miRNA) are transcribed by RNA polymerase II or III. These transcripts are subsequently cleaved by the microprocessor complex DROSHA-DGCR8 to form the pre-miRNA, which is transported from the nucleus to the cytoplasm by XPO5-RAN-GTP. There, it is cleaved by DICER1-TRBP to form the two stranded miRNA duplex; the passenger strand is detached and normally degraded, although in some cases it acts as a separate functional miRNA. The remaining functional strand combines with E1F2C2 proteins and forms the RNA-induced silencing complex (RISC). The miRNA then guides RISC to prevent translation of target mRNAs. Translation is prevented by mRNA deadenylation, mRNA target cleavage or translational repression [11]. Of the mechanisms of posttranscriptional regulation by miRNAs, lowered mRNA levels (mRNA cleavage or deadenylation) accounts for most ( $>84 \%$ ) of decreased protein production [12]. This implies that it is possible to assess levels of miRNA mediated gene silencing from the mRNA levels of a miRNA's target transcripts.

Cheng et al. quantified miRNA activity in this way by defining the regulatory effect score (RE-score) of a miRNA in a sample as the average expression rank of genes that are not predicted to be targeted by the miRNA minus the average expression rank of the predicted targets of the miRNA [13]. Thus, the RE-score is intended to measure the extent to which targets of the miRNA are downregulated in a sample relative to other genes. It is not informative to compare the RE-scores of different miRNAs, but comparison of the RE-score of a given miRNA between samples can provide an indication of a difference in the repressive effect of the miRNA in the samples. For example, if the targets of a given miRNA relative to nontargets are ranked higher in a set of cancer samples than in comparable normal tissues, this suggests that the miRNA exerts less control over gene expression in the cancer samples. There have been numerous other studies published that have also investigated miRNA regulation by assessing changes in expression of mRNA targets [14-18].

We sought to investigate whether there is evidence of natural variation in this phenotype between human individuals using RE-scores calculated from microarray and
RNA-seq data generated from the CEU (Utah residents with ancestry from northern and western Europe) and YRI (Yoruba in Ibadan, Nigeria) lymphoblastoid cell lines of the HapMap project [19-23]. Microarray data were available for 56 trios of related individuals in these populations (consisting of two parents and an offspring). We used these data to investigate the genetic component of the variation in RE-scores. Positive correlation between the value of a phenotype in an offspring and the mean value in parents provides evidence of a heritable component in the variation of the phenotype and the slope of the linear regression line relating parent mean to offspring values can be used as an estimate of the narrow-sense heritability [24-26].

\section{Results and discussion}

\section{Heritability of the regulatory effect of miRNAs}

Microarray data [23] were obtained for 56 trios (both parents and an offspring) from the CEU and YRI populations of the HapMap project $[19,20]$. Using miRNA targets predicted by TargetScan $[2,27]$ we compared REscores between parents and offspring. For $51 \%$ of miRNAs the mean RE-score of parents and the RE-score of the offspring were significantly $(p<0.05)$ positively correlated (Table 1). Population of origin was included in these regressions to model biological and technical differences between the CEU and YRI cell lines. Regression p-values and slopes for heritability of individual miRNA RE-scores from TargetScan and a second miRNA prediction algorithm (PicTar [28]) are provided as Additional file 1; histograms of these p-values are shown in Additional file 2: Figure S1.

We calculated the mean of the RE-score over all miRNAs. Unsurprisingly, the mean RE-score is also strongly correlated between parents and offspring in HapMap trios (Figure 1). This correlation is statistically significant using mean RE-scores calculated from targets predicted by TargetScan (slope $=0.68 \pm 0.34 ; p=2 \times 10^{-4}$ ). The slopes of these regression lines provide estimates of the narrow-sense heritability of the mean RE-score. We also assessed mean RE-score heritability based on targets predicted by four other algorithms (which have been found to

\section{Table 1 Summary of results for individual miRNA RE-scores calculated using TargetScan}

\begin{tabular}{lc}
\hline Number of miRNAs & 244 \\
\hline Average number of target genes per miRNA & 437 \\
\hline RE-score positively correlated between & 235 \\
mean of parent and offspring & 124 \\
\hline Positively correlated $(p<0.05)$ & $0.30(0.15)$ \\
\hline
\end{tabular}




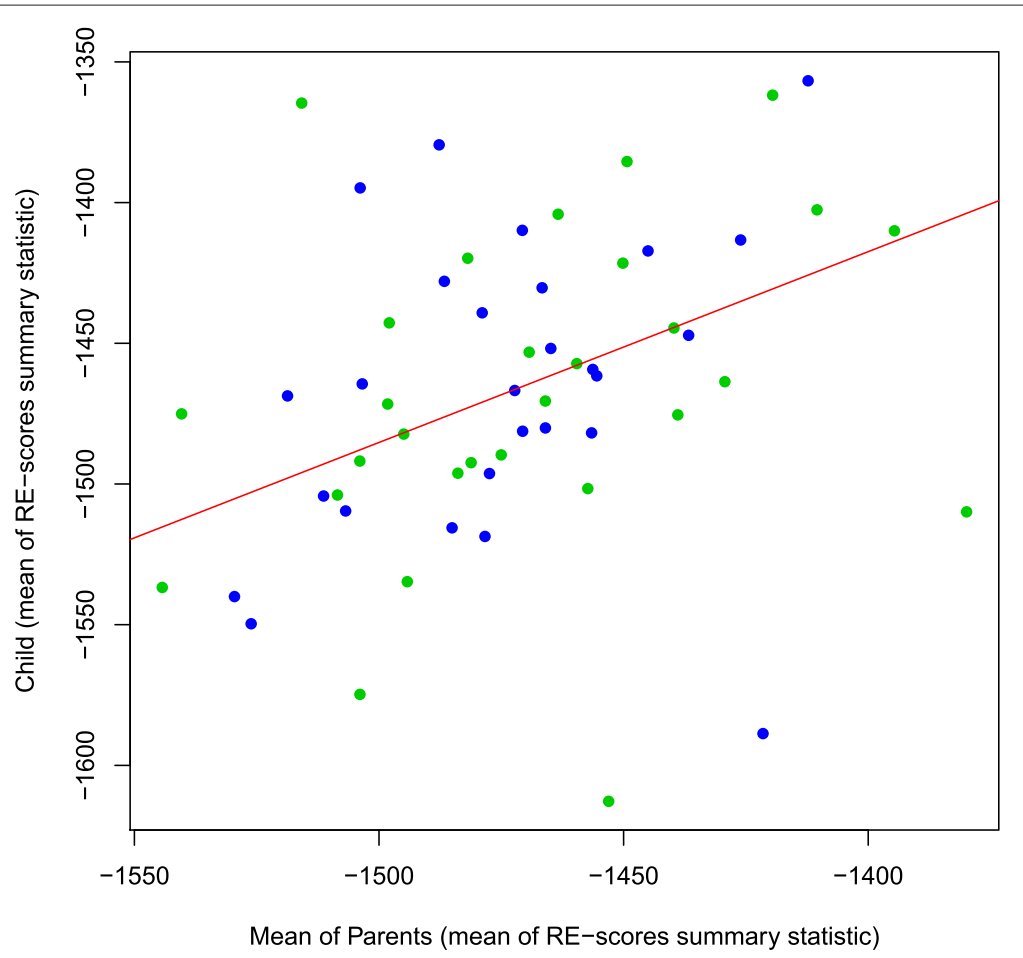

Figure 1 Heritability of mean RE-score. Scatter plots of child values of mean RE-score against mean value of parents. Points from the CEU are colored blue and YRI are green. The estimated regression line is shown in red. RE-scores were calculated using TargetScan.

be less accurate predictors of protein levels [29]). Of these PicTar $\left(\right.$ slope $=0.62 \pm 0.36 ; p=1.3 \times 10^{-3}$ ), miRanda $[30]$ (slope $\left.=0.40 \pm 0.37 ; p=3.6 \times 10^{-2}\right)$ and mirTarget2 [31] (slope $=0.35 \pm 0.32 ; p=2.8 \times 10^{-2}$ ) showed significant evidence of heritability, while one miRNA target prediction algorithm, mirBase [32], did not reach statistical significance $($ slope $=0.20 \pm 0.33 ; p=0.21$ ).

It is possible that the apparent genetic contribution to the regulatory effect of miRNAs is a consequence of the heritability of gene expression, rather than a novel molecular phenotype. Since the expression levels of a large proportion of human genes have a strong genetic component [33-35], the correlation in RE-score between parents and offspring could simply reflect the correlation in the expression levels of a proportion of the genes targeted by the miRNA. We devised a permutation test to evaluate this possibility. For each set of mRNAs predicted to be targeted by a given miRNA we replaced predicted target genes by genes chosen at random (details in Methods). If the apparent heritability of RE-scores is merely a consequence of heritability of individual gene expression levels, the RE-scores obtained from sets of random genes should exhibit similar levels of heritability to the RE-scores based on the true predicted target sets. Greater evidence of heritability from true predicted targets compared to sets of randomly selected genes suggests that the RE-score heritability cannot be explained by the heritability of individual gene expression levels. Of 1,000 randomizations, just eight $(p=0.008)$ reached a regression p-value as extreme as the target sets predicted by TargetScan.

\section{Genome-wide association of mean RE-score}

In order to explore the genetic contribution to RE-score variation further, we carried out a genome-wide association (GWA) test, treating mean RE-score, calculated using miRNA targets predicted by TargetScan, as a quantitative trait, and using genotype data from the HapMap project $[19,20]$. To avoid artifacts resulting from population structure, we carried out these tests separately on the CEU and YRI samples and excluded related individuals (offspring of the HapMap trios). RE-scores were recalculated using expression data derived from RNA-seq [21,22], which was available for parents but not for offspring of HapMap trios. Histograms and Manhattan plots of p-values are shown in Additional file 2: Figure S2. The p-value distributions show a peak towards low p-values, suggesting the presence of some true positive associations. The top ten most significantly associated loci in both populations are shown in Additional file 3. None of these associations remained significant following a permutation-based correction for multiple testing. This is not surprising given the relatively small number of samples compared to typical GWA studies. 


\section{Association of mean RE-score with SNPs in the miRNA biogenesis pathway}

Cheng et al. [13] used the RE-score metric to compare miRNA repression in Estrogen Receptor Positive (ER+) and Estrogen Receptor Negative (ER-) breast cancers and found that miRNAs tended to have higher RE-scores (i.e. their targets were more repressed) in the latter. The differences in RE-scores between the two cancer subtypes was attributed to dysregulation of key genes in the microRNA biogenesis pathway [13]. We used linear regression to investigate the relationships between seven key genes in the miRNA biogenesis pathway, (DICER1, EIF2C2, DROSHA, DGCR8, XPO5, RAN and TRBP) and mean REscore, first using all samples from both populations pooled (including population of origin as a factor in the model) and then in each of the populations separately. Expression levels of five of these seven genes were significantly correlated with mean RE-score (Table 2), consistent with a contribution of differential regulation of the miRNA biogenesis pathway to differences in mean RE-score. In fact, a large proportion (37.8\%) of all genes were significantly associated $(p<0.05)$ with mean RE-score; however, this proportion was somewhat higher (five out of seven, or 71.4\%) for genes in the miRNA biogenesis pathway. Given this relationship between RE-score and the activities of genes in the miRNA biogenesis pathway these genes are worthy of closer examination for genetic association with mean RE-score.

We carried out a second test of association, restricting to 336 SNPs that map to the genomic regions of these seven key genes involved in the miRNA biogenesis pathway. A SNP is mapped to the genomic region of a gene by dbSNP if it lies between $2 \mathrm{~kb}$ upstream and $500 \mathrm{bp}$ downstream of the gene. Again there appear to be more low p-values than would be expected under the uniform distribution, pointing to a proportion of true positive associations in both populations (Additional file 2: Figure S3). The ten SNPs most strongly associated with mean RE-score in CEU and YRI are shown in Tables 3 and 4, respectively. One SNP, rs17409624, in an intron of DROSHA remained statistically significantly ( $p_{\text {adjusted }}<$ 0.05) associated with mean RE-score in the CEU following Bonferroni and permutation-based control for multiple testing. This SNP was also nominally significantly associated with mean RE-score in the YRI ( $p=0.04)$; however, the minor allele frequency was much lower in YRI, limiting the power to detect an association with a significance that could survive multiple test correction. The magnitude and direction of the RE-score differences between genotypes are consistent across the two populations (Figure 2). Taken individually, the vast majority (214 of 244) of RE-scores are associated $(p<0.05)$ with genotype at this SNP in the CEU. This number drops to 36 of 244 in the YRI, however the lower minor allele frequency in YRI again limits the power to detect the association.

As a further test of the association between rs17409624 and mean RE-score, we investigated the RE-scores of a particular class of intronic miRNAs (mirtrons), which are not processed by DROSHA [36]. If the association between the SNP and mean RE-score is real and is mediated by an effect on miRNA processing by $D R O S H A$, the SNP should not be associated with the RE-scores of mirtrons. Consistent with this prediction, we found that a much lower proportion of mirtron RE-scores (based on TargetScan predictions from CEU RNA-seq data) are associated (at $\alpha=0.05$ ) with the DROSHA SNP ( 5 out of 12 mirtrons, compared to 214 out of 244 conventional miRNAs; $p=0.0004$, from a two-sided Fisher's exact test). We have found evidence that the subset of mirtrons that do show an association with the SNP do so because of an overlap between their target gene sets and the target gene sets of conventional miRNAs, as the mirtrons which are most significantly associated with rs17409624 tend to target genes that are also targeted by many other miRNAs; and mirtrons that target genes that are targeted by few conventional miRNAs are less significantly associated with rs17409624 (Additional file 2: Figure S4).

Table 2 Associations between expression levels of key miRNA biogenesis genes and mean RE-score

\begin{tabular}{|c|c|c|c|c|c|c|}
\hline & \multicolumn{2}{|c|}{ CEU } & \multicolumn{2}{|c|}{ YRI } & \multicolumn{2}{|c|}{ Pooled } \\
\hline & Bonferroni P & Slope & Bonferroni P & Slope & Bonferroni P & Slope \\
\hline DROSHA & $9.42 \times 10^{-03}$ & -10.23 & $1.37 \times 10^{-05}$ & -22.12 & $5.19 \times 10^{-06}$ & -15.64 \\
\hline DGCR8 & 0.036 & 11.57 & 0.95 & -0.46 & 0.37 & 6.23 \\
\hline XPO5 & 0.47 & -3.03 & $1.38 \times 10^{-04}$ & -17.85 & $2.17 \times 10^{-03}$ & -10.74 \\
\hline RAN & 0.27 & 0.49 & 0.14 & -0.94 & 0.75 & -0.12 \\
\hline DICER1 & $8.51 \times 10^{-03}$ & -13.77 & $1.97 \times 10^{-09}$ & -26.18 & $5.57 \times 10^{-10}$ & -21.72 \\
\hline TRBP & $2.95 \times 10^{-05}$ & 12.26 & 0.085 & 8.12 & $2.68 \times 10^{-04}$ & 10.60 \\
\hline EIF2C2 & 0.022 & -6.25 & $1.39 \times 10^{-07}$ & -9.07 & $1.88 \times 10^{-08}$ & -8.41 \\
\hline
\end{tabular}

P-values and slopes from the linear regression of expression level of genes in the miRNA biogenesis pathway against mean RE-score, in the CEU, YRI and for both populations pooled. 
Table 3 Top 10 associations for miRNA biogenesis pathway related SNPs (CEU)

\begin{tabular}{|c|c|c|c|c|c|c|}
\hline & Location & Associated Gene & P-value & Bonferroni P & Q-value & $\begin{array}{c}\text { Permutation } \\
\text { p-value }\end{array}$ \\
\hline rs17409624 & chr5:31,528,733 & DROSHA & $1.81 \times 10^{-04}$ & 0.043 & 0.018 & 0.03 \\
\hline rs10078886 & chr5:31,427,441 & DROSHA & $3.32 \times 10^{-04}$ & 0.079 & 0.018 & 0.051 \\
\hline rs16901121 & chr5:31,418,215 & DROSHA & $3.32 \times 10^{-04}$ & 0.079 & 0.018 & 0.051 \\
\hline rs2279797 & chr5:31,428,028 & DROSHA & $3.32 \times 10^{-04}$ & 0.079 & 0.018 & 0.051 \\
\hline rs13183642 & chr5:31,511,106 & DROSHA & $1.25 \times 10^{-03}$ & 0.3 & 0.054 & 0.16 \\
\hline rs3805516 & chr5:31,420,670 & DROSHA & $1.56 \times 10^{-03}$ & 0.37 & 0.056 & 0.2 \\
\hline rs4867349 & chr5:31,536,327 & DROSHA & $1.82 \times 10^{-03}$ & 0.43 & 0.056 & 0.23 \\
\hline rs 2287584 & chr5:31,423,007 & DROSHA & $3.27 \times 10^{-03}$ & 0.78 & 0.073 & 0.33 \\
\hline rs615344 & chr5:31,425,788 & DROSHA & $3.39 \times 10^{-03}$ & 0.8 & 0.073 & 0.34 \\
\hline rs682902 & chr5:31,423,694 & DROSHA & $3.39 \times 10^{-03}$ & 0.8 & 0.073 & 0.34 \\
\hline
\end{tabular}

The results for association of miRNA biogenesis pathway related SNPs with mean RE-score in the CEU.

\section{Searching for causal SNPs}

We investigated the function of SNP rs17409624 using the "SNP Function Prediction" tool, which is part of the SNPinfo suite (available at http://www.niehs.nih.gov/ snpinfo) [37]; however, no significant results were identified. We also searched the "GWAS Catalog" but did not find any previous studies which had identified this SNP [38]. To search for other SNPs that may be causally responsible for this association we used confidence intervals [39] as implemented in HaploView to calculate haplotype blocks for the CEU HapMap data. rs17409624 is located within a haplotype block that includes the $D R O S H A$ promoter region (Figure 3 ). We verified that this is the active promoter of DROSHA using data recently released by the ENCODE project et al. [40]. Chromatin states for this locus are shown in Additional file 2: Figure S5. The expression level of DROSHA is significantly associated with mean RE-score (Table 2); however, the genotype of this locus was not significantly correlated with DROSHA expression level ( $\mathrm{p}=0.39$ ) or with the relative expression level of any DROSHA transcript isoforms (identified using Cufflinks [41]). A further possibility is that rs17409624 is in linkage disequilibrium (LD) with an exonic SNP that was not genotyped on the HapMap microarrays. Using SNP calls from genome sequence data released by the 1,000 Genomes Project [42] we found no coding SNPs with a stronger association to mean REscore than rs17409624, the regions assayed included the $3^{\prime}$ and $5^{\prime}$ UTRs. We caution however, that there was much less statistical power to detect an association using the 1,000 Genomes data, as there was an overlap of only 45 samples between the 1,000 Genomes Project dataset (versus 59 for the HapMap microarray data) and the RNAseq samples from the CEU used in this analysis, which means that it is difficult to rule out the possibility of linkage of rs17409624 with a causative SNP in the coding region. These results are provided in Additional file 4. Thus, the causal mechanism linking genetic variation

Table 4 Top 10 associations for miRNA pathway related SNPs (YRI)

\begin{tabular}{|c|c|c|c|c|c|c|}
\hline & Location & Associated Gene & P-value & Bonferroni P & Q-value & $\begin{array}{c}\text { Permutation } \\
\text { p-value }\end{array}$ \\
\hline rs6994531 & chr8:141,544,476 & EIF2C2 & $4.57 \times 10^{-03}$ & 1 & 0.38 & 0.55 \\
\hline rs1633445 & chr22:20,100,596 & DGCR8 & 0.011 & 1 & 0.38 & 0.77 \\
\hline rs17409275 & chr5:31,514,127 & DROSHA & 0.012 & 1 & 0.38 & 0.8 \\
\hline rs1209904 & chr14:95,563,712 & DICER1 & 0.015 & 1 & 0.38 & 0.86 \\
\hline rs1187650 & chr14:95,551,554 & DICER1 & 0.018 & 1 & 0.38 & 0.9 \\
\hline rs1187655 & chr14:95,565,556 & DICER1 & 0.018 & 1 & 0.38 & 0.9 \\
\hline rs6575499 & chr14:95,622,200 & DICER1 & 0.018 & 1 & 0.38 & 0.9 \\
\hline rs12881840 & chr14:95,568,003 & DICER1 & 0.020 & 1 & 0.38 & 0.9 \\
\hline rs12889800 & chr14:95,618,898 & DICER1 & 0.020 & 1 & 0.38 & 0.9 \\
\hline rs2292780 & chr8:141,561,357 & EIF2C2 & 0.022 & 1 & 0.38 & 0.93 \\
\hline
\end{tabular}



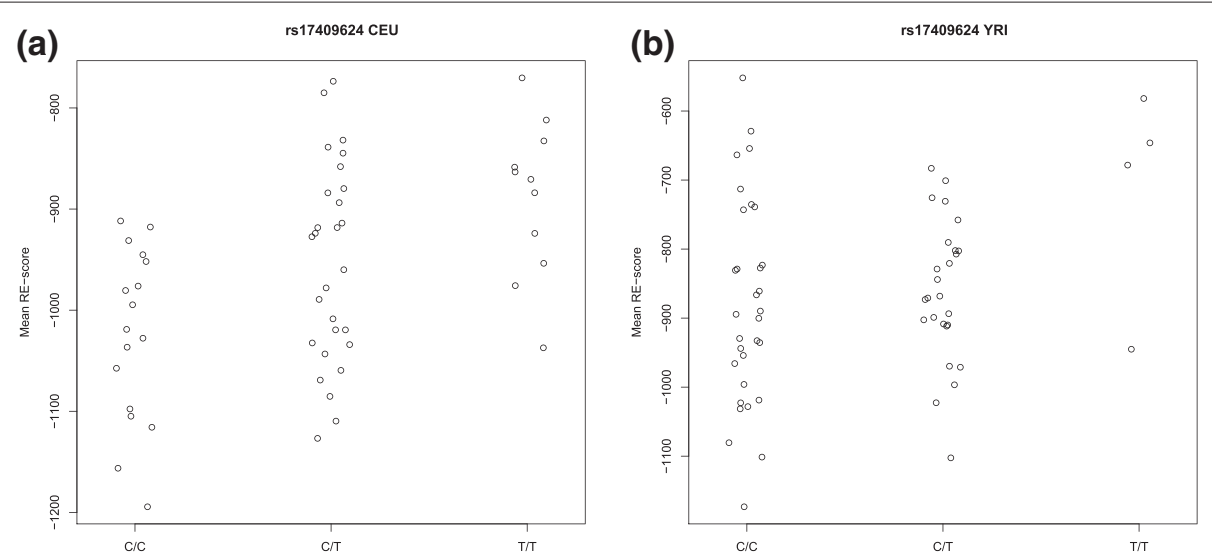

Figure 2 Stripcharts for rs 17409624. Stripcharts of mean RE-score against genotype at rs17409624 in the (a) CEU and (b) YRI populations.

at the DROSHA locus to variation in the RE-score remains unclear.

Integrative analysis of miRNA expression and RE-score data miRNA expression data has recently been generated for some of the HapMap CEU and YRI cell lines [43]. In the majority of cases, miRNA expression levels and their corresponding RE-scores were not significantly correlated. Average Spearman correlation between miRNA expression and corresponding TargetScan based RE-score from the RNA-seq data is only 0.009 in the CEU and -0.0003 in the YRI. Although surprising, this observation is consistent with the findings of Cheng et al. [13], who, for the original RE-score study, performed Spearman

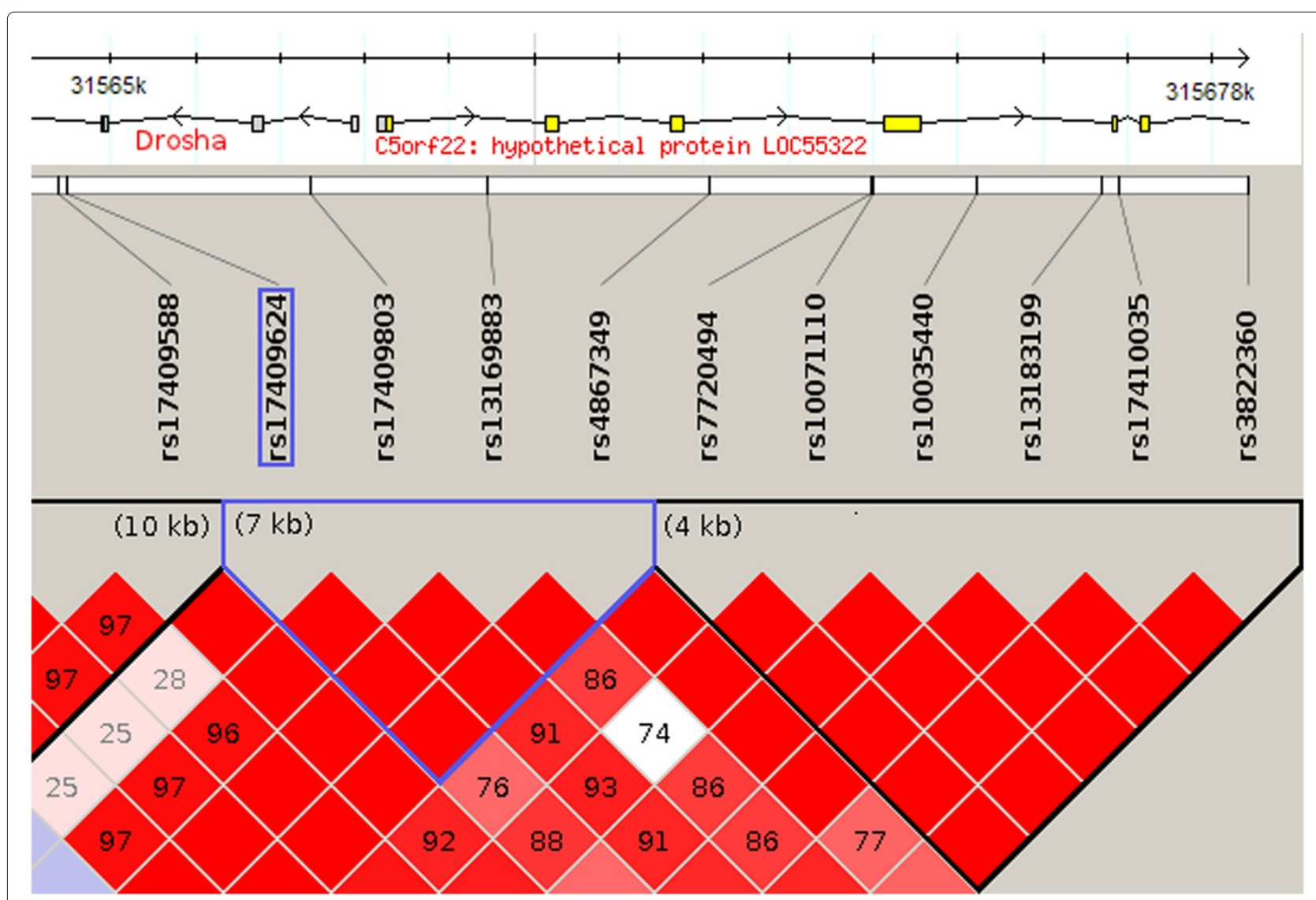

Figure 3 Haplotypes in rs 17409624 region. Haplotype blocks around rs 17409624 as calculated by confidence intervals in Haploview, using the HapMap CEU data. The block which includes rs 17409624 is highlighted in blue; this block also includes the DROSHA promoter region. 
correlations of the $t$-scores of comparisons of miRNA expression and RE-scores between ER- and ER+ breast cancers, finding only very weak positive correlation. Similar results have also been observed on two separate datasets by Liang et al. [44]. Correlations between miRNA expression level and RE-scores are included in Additional file 5. However, we find that in the CEU, the expression of 17 of 201 miRNAs that were consistently expressed across the cell lines is associated $(p<0.05)$ with rs17409624 and that 13 of these associations are in the same direction as mean RE-score. One miRNA is associated with the SNP in the YRI, but once again, the lower minor allele frequency of rs17409624 in the YRI limits the power to identify associations. P-values and false discovery rates for these 18 miRNAs (17 CEU and 1 YRI) for genotype association are included in Additional file 6. Thus, this SNP represents a trans-eQTL cluster for miRNA gene expression. We hypothesize that this trans-eQTL reflects inter-individual differences in the efficiency of miRNA processing by DROSHA. Given that miRNA expression measurements are relative (in this case miRNA expression was measured using a pooled reference microarray design), it is possible that this polymorphism may affect the absolute copy numbers of a large fraction of miRNAs, even though an association between miRNA expression and the SNP is detectable for a relatively small fraction of miRNAs. This hypothesis could be tested using transcriptome sequencing strategies designed to measure the abundance of miRNAs relative to other RNA species. Indeed, given a global and consistent change in expression of all miRNAs in a sample, one may not expect the expression of any miRNAs to be associated with rs17409624, as the proportion of the transcript pool occupied by any given miRNA, would remain unchanged. However, the miRNA regulatory effect polymorphism need not affect the expression of all miRNAs to exactly the same degree, potentially leading to both positive and negative associations of miRNA expression with the SNP.

As discussed above, RE-scores of the majority of miRNAs were not correlated with miRNA expression. This remained the case when we restricted to miRNAs whose expression varied most across samples. However, the RE-scores of individual miRNAs were correlated with the mean RE-score calculated across all miRNAs. We restricted this analysis to the 20 most variable miRNAs. Of the top 20 in either population, 14 in the CEU and 13 in the YRI had TargetScan prediction data and therefore RE-scores. We only considered these highly variable miRNAs because quantities that are relatively constant across samples are not expected to be correlated, given the noise inherent in microarray data. The correlation between mean and individual miRNA RE-scores is not simply a consequence of overlaps in genes targeted by different miRNAs, since it holds true even when the mean
RE-score is recalculated, for each miRNA correlation test, after all of the individual miRNAs targets have been subtracted from the target sets of the remaining miRNAs. 13 of the 14 highly varying miRNAs in the CEU and all 13 of 13 in the YRI show a stronger association between the individual RE-score and (subtracted) mean RE-score, than between the individual RE-score and the expression of the miRNA itself. In most cases this difference is large (Additional file 7), hence, the mean RE-score in a sample may be a much better predictor of the expression level of the targets of any particular miRNA, than is the expression profile of the miRNA itself. It is, perhaps, not surprising that the expression level of an individual miRNA is not indicative of the expression of its target genes, given that targeted genes are often targets of a large number of miRNAs. Of 11,759 genes which are predicted to be targeted by at least one miRNA (by the full TargetScan set), the average number of miRNAs targeting each gene is 17.48. In this context, the fact that the mean RE-score has power to predict the expression levels of a miRNA target, even when the mean RE-score is calculated without considering the targets of that miRNA is interesting and points to differences in the effect of the miRNA pathway on target genes across the cell lines.

\section{Conclusions}

We have found evidence of heritability of the regulatory effect of miRNAs in human. We have also identified an association between the regulatory effect of miRNAs and a SNP in the miRNA processing gene DROSHA. This association was identified in lymphoblastoid cell lines and it remains to be seen whether and in which primary cells the regulatory effect of miRNAs is associated with the DROSHA locus. As noted in the Background, Cheng et al. had observed that there is a change in miRNA RE-scores between ER- and ER+ breast cancer subtypes. Thomsom et al. showed that mature miRNA levels are generally lower in several human primary cancers, despite unchanged pri-miRNA levels and this has been attributed to defective processing by DROSHA [45], while DROSHA and DICER have also been shown to be downregulated in endometrial cancer and specific subgroups of breast cancer $[46,47]$. Thus, it will be important to investigate further the phenotypic consequences of inter-individual differences in miRNA regulatory efficiency and the influence on gene expression, possible tumorigenesis and the impact of such inter-individual differences in the context of the use of miRNAs as biomarkers.

\section{Methods \\ Data}

Raw gene expression microarray data of related individuals from the CEU and YRI populations of the HapMap 
project were downloaded from GEO under accession number GSE7792, these data were generated by Huang et al. [23] using Affymetrix Human Exon 1.0 ST microarrays. Prior to calculating gene expression level estimates, the data were RMA normalized [48] and genes whose expression level were below the detection threshold, as estimated by the DABG algorithm $(p<0.05)$, were set to zero; these steps were performed using Affymetrix Power Tools and R as described in [49]. RNA-seq data for unrelated individuals of the HapMap YRI population were generated by Pickrell et. al [21] and we obtained these aligned data from GEO under accession number GSE19480. Similarly, Montgomery et al. [22] used RNA-seq to assess gene expression of unrelated CEU samples and these data were obtained from ArrayExpress under accession number E-MTAB-197. All data were aligned to $h g 18$ using MAQ [50]. We performed gene expression analysis using $\mathrm{R} /$ Bioconductor. Data were loaded in R [51] using the ShortRead [52] library. Following Montgomery et al., only reads that had a mapping quality score of greater than or equal to 10 were included. The GenomicRanges [53] library was used to compute the number of reads mapping to exons of each gene and expression values were normalized using the RPKM [54] procedure. miRNA prediction data were obtained using the $\mathrm{R}$ library RmiR.Hs.miRNA [55] which provides a database of miRNA targets for several widely used algorithms. The HapMap release 28 (merged data for phases I, II and III) $[19,20]$ SNP data were downloaded from the HapMap website, converted to GenABEL format and trimmed to include only samples in the CEU and YRI populations for which there was matching RNA-seq data.

\section{Estimating Heritability of mean RE-score}

Narrow sense heritability of individual miRNA RE-scores and mean RE-score was estimated using a robust linear regression model $[24,25]$. The $r \operatorname{lm}()$ function from the $\mathrm{R}$ library MASS was used to fit regression models for child value dependent on mean of parents. Population of origin was included as a factor in the models. The slope of the regression line provides an estimate of heritability.

\section{Permutation testing of heritability of mean RE-score}

To calculate a corrected p-value for heritability of mean RE-score of a miRNA prediction algorithm, we performed 1,000 permutations of the prediction algorithm's miRNA gene target sets and recalculated heritability of mean RE-score following each permutation; the permutation $\mathrm{p}$ value was the proportion of permuted sets that return $\mathrm{p}$-values which are equal to, or lower than, the original raw $\mathrm{p}$-value for that algorithm. To perform a permutation, we replace each gene target of each miRNA's target set with a randomly chosen gene, but only genes for which expression data is available are replaced or used for replacement, as only these can affect RE-scores. If a gene is a target of multiple microRNAs, it is replaced by the same randomly chosen gene in every target set, so as to maintain the structure of the data.

\section{Genome-wide association test}

The R package GenABEL [56,57] was used for filtering and tests of association. Prior to testing for association, genotype data were filtered as follows. Obvious close relatives are removed by discarding the child samples and to avoid the effects of population stratification CEU and YRI samples are assayed separately. Markers with a low minor allele frequency were filtered by excluding SNPs for which there were less than 5 copies of the minor allele across all samples. We used only SNPs genotyped as part of HapMap phase III. Individuals or SNPs were excluded for a call rate of $<0.95$. Tests for HardyWeinberg equilibrium were conducted using Pearson's $\chi^{2}$, comparing observed genotype frequencies in the data to the calculated expected frequencies; a cut-off FDR level of 0.2 was applied. To assess if any remaining relatedness exists among the samples, the pairwise proportion of alleles identical-by-state (IBS) was calculated between all individuals based on 2,000 randomly chosen autosomal markers, ensuring IBS $<0.95$ for all samples. For multiple testing correction of association p-values, permutations were calculated by permuting phenotype labels and performing tests of association as normal; for each raw $\mathrm{p}$-value, we computed the number of permutations for which a $\mathrm{p}$-value equal to, or lower than, the original raw $\mathrm{p}$-value was reached and divide this by the number of permutations, the result of which is the adjusted pvalue. False discovery rates were also assessed using the $R$ package qvalue [58].

\section{Calculating association between individual miRNA RE-score, mean RE-score and miRNA expression}

For each of 14 highly varying miRNAs in the CEU samples and 13 in the YRI, we fit a multiple linear regression model of individual miRNA RE-score dependent on the expression of the miRNA and the mean RE-score. For each fit of the model, mean RE-score was re-calculated with the genes that are targets of the particular individual miRNA removed from the gene expression matrix, so as to avoid a bias in the association between the two variables.

\section{Additional files}

Additional file 1: Tables of regression p-values and slopes, for heritability of individual miRNA RE-scores from TargetScan and PicTar algorithms. 
Additional file 2: Figure S1. Heritability for individual RE-scores. Histograms of $p$-values for tests of heritability of individual RE-scores for (a) TargetScan and (b) PicTar algorithms. Figure S2: P-Values for genome-wide tests of association. Histograms ( $\&$ \& b) of p-values for tests of association between all SNP markers and mean RE-score and Manhattan plots (c \& d) of p-values in the CEU and YRI respectively. Figure S3: Histograms of $p$-values for miRNA biogenesis pathway SNPs. Histograms of $p$-values for the tests of association between SNP markers mapped to the miRNA biogenesis pathway and mean RE-score in the (a) CEU and (b) YRI populations. Figure S4: Many mirtron target genes are also miRNA targets Relationship between the strength of association with rs 17409624 for mirtrons and the average number of conventional miRNAs that also target the mirtron's target genes. This figure is based on TargetScan predictions for conserved miRNA families on HapMap CEU data. $R^{2}=0.65$, $p=5.1 \times 10^{-4}$ Figure S5: DROSHA promoter region Chromatin state of DROSHA region for nine cell lines from the ENCODE project. Active promoter is shown in bright red. The haplotype block for rs 17409624 is shown in black and clearly overlaps the promoter region.

Additional file 3: Tables of top 10 SNPs from the genome-wide screen for association of mean RE-score and SNP genotypes, in the CEU and in the YRI.

Additional file 4: Table of $p$-values for association of Drosha coding SNPs and mean RE-score, based on the 1,000 genomes CEU data. Only SNPs with more than 1 copy of the minor allele in the 45 samples available are included. If a SNP was genotyped by the HapMap project, that p-value is also included.

Additional file 5: Tables of Spearman correlations and $p$-values for correlations between individual miRNAs and their associated RE-scores in the CEU and YRI.

Additional file 6: Tables of $\mathrm{P}$-values and False discovery rates (calculated using the qvalue package in $\mathrm{R}$ ) for association between the expression of individual miRNAs and rs 17409624. MiRNAs with $p<0.05$ are shown.

Additional file 7: P-values from linear models of individual RE-score dependent on (subtracted) mean RE-score and miRNA expression.

\section{Competing interests}

The authors declare that they have no competing interests.

\section{Author's contributions}

PG performed the analysis and drafted the manuscript. CS conceived the study. CS and AG directed the project. RSH and ERG generated the miRNA expression data and provided guidance on analysis. All authors read and approved the final manuscript.

\section{Acknowledgements}

PG is supported by the Irish Research Council for Science, Engineering and Technology (IRCSET) Embark Initiative's Enterprise Partnership Scheme. RSH receives support from $\mathrm{NIH} / \mathrm{NCl}$ grant R21 CA139278, NIH/NIGMS grant K08GM089941, University of Chicago Cancer Center Support Grant (\#P30 CA14599) and Breast Cancer SPORE Career Development Award. CS is supported by Science Foundation Ireland (07/SK/M1211b).

\section{Author details}

${ }^{1}$ Department of Mathematics, Statistics and Applied Mathematics, National University of Ireland, Galway, Ireland. ${ }^{2}$ Section of Hematology/Oncology, Department of Medicine, University of Chicago, Chicago, USA. ${ }^{3}$ Section of Genetic Medicine, Department of Medicine, University of Chicago, Chicago, USA. ${ }^{4}$ Department of Genetics, Albert Einstein College of Medicine, 1300 Morris Park Ave, Bronx, NY 10461, USA. ${ }^{5}$ Institute of Infectious Disease and Molecular Medicine, University of Cape Town, Cape Town 7925, South Africa.

Received: 16 January 2012 Accepted: 30 July 2012

Published: 10 August 2012

\section{References}

1. Krol J, Loedige I, Filipowicz W: The widespread regulation of microRNA biogenesis, function and decay. Nat Rev Genet 2010, 11(9):597-610. [http://dx.doi.org/10.1038/nrg2843]
2. Grimson A, Farh KKH, Johnston WK, Garrett-Engele P, Lim LP, Bartel DP: MicroRNA targeting specificity in mammals: determinants beyond seed pairing. Mol Cell 2007, 27:91-105. [http://dx.doi.org/10.1016/j. molcel.2007.06.017]

3. Chekulaeva M, Filipowicz W: Mechanisms of miRNA-mediated post-transcriptional regulation in animal cells. Curr Opin Cell Bio/ 2009 21(3):452-460. [http://dx.doi.org/10.1016/j.ceb.2009.04.009]

4. Bartel DP: MicroRNAs: target recognition and regulatory functions. Cell 2009, 136(2):215-233. [http://dx.doi.org/10.1016/j.cell.2009.01.002]

5. Xu P, Guo M, Hay BA: MicroRNAs and the regulation of cell death. Trends Genet 2004, 20(12):617-624. [http://dx.doi.org/10.1016/j.tig.2004. 09.010]

6. Cheng AM, Byrom MW, Shelton J, Ford LP: Antisense inhibition of human miRNAs and indications for an involvement of miRNA in cell growth and apoptosis. Nucleic Acids Res 2005, 33(4):1290-1297. [http:// dx.doi.org/10.1093/nar/gki200]

7. Visone R, Croce CM: MiRNAs and cancer. Am J Pathol 2009 174(4):1131-1138. [http://dx.doi.org/10.2353/ajpath.2009.080794]

8. Eis PS, Tam W, Sun L, Chadburn A, Li Z, Gomez MF, Lund E, Dahlberg JE: Accumulation of miR-155 and BIC RNA in human B cell lymphomas. Proc Natl Acad Sci U S A 2005, 102(10):3627-3632. [http://dx.doi.org/10. 1073/pnas.0500613102]

9. Cimmino A, Calin GA, Fabbri M, lorio MV, Ferracin M, Shimizu M, Wojcik SE, Aqeilan RI, Zupo S, Dono M, Rassenti L, Alder H, Volinia S, Liu CG, Kipps TJ, Negrini M, Croce CM: miR-15 and miR-16 induce apoptosis by targeting BCL2. Proc Natl Acad Sci U S A 2005, 102(39):13944-13949. [http://dx.doi.org/10.1073/pnas.0506654102]

10. Calin GA, Croce CM: MicroRNAs and chromosomal abnormalities in cancer cells. Oncogene 2006, 25(46):6202-6210. [http://dx.doi.org/10. 1038/sj.onc.1209910]

11. Winter J, Jung S, Keller S, Gregory RI, Diederichs S: Many roads to maturity: microRNA biogenesis pathways and their regulation. Nat Cell Biol 2009, 11(3):228-234. [http://dx.doi.org/10.1038/ncb0309-228]

12. Guo H, Ingolia NT, Weissman JS, Bartel DP: Mammalian microRNAs predominantly act to decrease target mRNA levels. Nature 2010, 466:835-840.

13. Cheng C, Fu X, Alves P, Gerstein M: mRNA expression profiles show differential regulatory effects of microRNAs between estrogen receptor-positive and estrogen receptor-negative breast cancer. Genome Bio/ 2009, 10(9):R90 [http://dx.doi.org/10.1186/gb-2009-10-9-r90]

14. Yu Z, Jian Z, Shen SH, Purisima E, Wang E: Global analysis of microRNA target gene expression reveals that miRNA targets are lower expressed in mature mouse and Drosophila tissues than in the embryos. Nucleic Acids Res 2007, 35:152-164. [http://dx.doi.org/10.1093/ nar/gkl1032]

15. Cheng C, Li LM: Inferring microRNA activities by combining gene expression with microRNA target prediction. PLOS One 2008 , 3(4):e1989. [http://dx.doi.org/10.1371/journal.pone.0001989]

16. Arora A, Simpson DA: Individual mRNA expression profiles reveal the effects of specific microRNAs. Genome Bio/ 2008, 9(5):R82. [http://dx.doi. org/10.1186/gb-2008-9-5-r82]

17. Wang $X$, Wang $X$ : Systematic identification of microRNA functions by combining target prediction and expression profiling. Nucleic Acids Res 2006, 34(5):1646-1652. [http://dx.doi.org/10.1093/nar/gkl068]

18. Buffa FM, Camps C, Winchester L, Snell CE, Gee HE, Sheldon H, Taylor M, Harris AL, Ragoussis J: microRNA associated progression pathways and potential therapeutic targets identified by integrated mRNA and microRNA expression profiling in breast cancer. Cancer Res 2011. [http://dx.doi.org/10.1158/0008-5472.CAN-11-0489]

19. Consortium $\mathrm{IH}$ : The International HapMap Project. Nature 2003 426(6968):789-796

20. International HapMap Consortium: A second generation human haplotype map of over 3.1 million SNPs. Nature 2007, 449(7164):851-861. [http://dx.doi.org/10.1038/nature06258]

21. Pickrell JK, Marioni JC, Pai AA, Degner JF, Engelhardt BE, Nkadori E, Veyrieras JB, Stephens M, Gilad Y, Pritchard JK: Understanding mechanisms underlying human gene expression variation with RNA sequencing. Nature 2010, 464(7289):768-772. [http://dx.doi.org/10. 1038/nature08872]

22. Montgomery SB, Sammeth M, Gutierrez-Arcelus M, Lach RP, Ingle C, Nisbett J, Guigo R, Dermitzakis ET: Transcriptome genetics using 
second generation sequencing in a Caucasian population. Nature 2010, 464(7289):773-777. [http://dx.doi.org/10.1038/nature08903]

23. Huang RS, Duan S, Bleibel WK, Kistner EO, Zhang W, Clark TA, Chen TX, Schweitzer AC, Blume JE, Cox NJ, Dolan ME: A genome-wide approach to identify genetic variants that contribute to etoposide-induced cytotoxicity. Proc Nat/ Acad Sci U S A 2007, 104(23):9758-9763. [http://dx. doi.org/10.1073/pnas.0703736104]

24. Visscher PM, Hill WG, Wray NR: Heritability in the genomics era-concepts and misconceptions. Nat Rev Genet 2008, 9(4):255-266. [http://dx.doi.org/10.1038/nrg2322]

25. Wray PN, Visscher: Estimating Trait Heritability. Nature Education 2008

26. Hamilton MB: Population Genetics: John Wiley \& Sons; 2009.

27. Lewis $B P$, Burge $C B$, Bartel DP: Conserved seed pairing, often flanked by adenosines, indicates that thousands of human genes are microRNA targets. Cell 2005, 120:15-20. [http://dx.doi.org/10.1016/j.cell. 2004.12.035]

28. Krek A, Grün D, Poy MN, Wolf R, Rosenberg L, Epstein EJ, MacMenamin P, da Piedade I, Gunsalus KC, Stoffel M, Rajewsky N: Combinatorial microRNA target predictions. Nat Genet 2005, 37(5):495-500. [http://dx. doi.org/10.1038/ng1536]

29. Baek D, Villén J, Shin C, Camargo FD, Gygi SP, Bartel DP: The impact of microRNAs on protein output. Nature 2008, 455(7209):64-71. [http:// dx.doi.org/10.1038/nature07242]

30. John B, Enright AJ, Aravin A, Tuschl T, Sander C, Marks DS: Human MicroRNA targets. PLoS Biol 2004, 2(11):e363. [http://dx.doi.org/10.1371/ journal.pbio.0020363]

31. Wang $X$, Naqa IME: Prediction of both conserved and nonconserved microRNA targets in animals. Bioinformatics 2008, 24(3):325-332. [http://dx.doi.org/10.1093/bioinformatics/btm595]

32. Griffiths-Jones S, Saini HK, van Dongen S, Enright AJ: miRBase: tools for microRNA genomics. Nucleic Acids Res 2008, 36(Database issue):D154-D158. [http://dx.doi.org/10.1093/nar/gkm952]

33. Cheung VG, Conlin LK, Weber TM, Arcaro M, Jen KY, Morley M, Spielman RS: Natural variation in human gene expression assessed in lymphoblastoid cells. Nat Genet 2003, 33(3):422-425. [http://dx.doi.org/ 10.1038/ng1094]

34. Schadt EE, Monks SA, Drake TA, Lusis AJ, Che N, Colinayo V, Ruff TG, Milligan SB, Lamb JR, Cavet G, Linsley PS, Mao M, Stoughton RB, Friend SH: Genetics of gene expression surveyed in maize, mouse and man Nature 2003, 422(6929):297-302. [http://dx.doi.org/10.1038/nature01434]

35. Brem RB, Yvert G, Clinton R, Kruglyak L: Genetic dissection of transcriptional regulation in budding yeast. Science 2002, 296(5568):752-755. [http://dx.doi.org/10.1126/science.1069516]

36. Berezikov E, Chung WJ, Willis J, Cuppen E, Lai EC: Mammalian mirtron genes. Mol Cell 2007, 28(2):328-336. [http://dx.doi.org/10.1016/j.molcel. 2007.09.028]

37. Xu Z, Taylor JA: SNPinfo: integrating GWAS and candidate gene information into functional SNP selection for genetic association studies. Nucleic Acids Res 2009, 37(Web Server issue):W600-W605. [http://dx.doi.org/10.1093/nar/gkp290]

38. Hindorff LA, Sethupathy P, Junkins HA, Ramos EM, Mehta JP, Collins FS, Manolio TA: Potential etiologic and functional implications of genome-wide association loci for human diseases and traits. Proc Natl Acad Sci U S A 2009, 106(23):9362-9367. [http://dx.doi.org/10.1073/ pnas.0903103106]

39. Gabriel SB, Schaffner SF, Nguyen H, Moore JM, Roy J, Blumenstiel B, Higgins J, DeFelice M, Lochner A, Faggart M, Liu-Cordero SN, Rotimi C, Adeyemo A, Cooper R, Ward R, Lander ES, Daly MJ, Altshuler D: The structure of haplotype blocks in the human genome. Science 2002, 296(5576):2225-2229. [http://dx.doi.org/10.1126/science.1069424]

40. Ernst J, Kheradpour P, Mikkelsen TS, Shoresh N, Ward LD, Epstein CB, Zhang X, Wang L, Issner R, Coyne M, Ku M, Durham T, Kellis M, Bernstein $B E$ : Mapping and analysis of chromatin state dynamics in nine human cell types. Nature 2011, 473(7345):43-49. [http://dx.doi.org/10. 1038/nature09906]

41. Trapnell C, Williams BA, Pertea G, Mortazavi A, Kwan G, van Baren MJ Salzberg SL, Wold BJ, Pachter L: Transcript assembly and quantification by RNA-Seq reveals unannotated transcripts and isoform switching during cell differentiation. Nat Biotechnol 2010, 28(5):511-515. [http://dx.doi.org/10.1038/nbt.1621]
42. Consortium GP: A map of human genome variation from population-scale sequencing. Nature 2010, 467(7319):1061-1073. [http://dx.doi.org/10.1038/nature09534]

43. Huang RS, Gamazon ER, Ziliak D, Wen Y, Im HK, Zhang W, Wing C, Duan S, Bleibel WK, Cox NJ, Dolan ME: Population differences in microRNA expression and biological implications. RNA Bio/ 2011, 8(4):692-701. [http://dx.doi.org/10.4161/rna.8.4.16029]

44. Liang Z, Zhou $\mathrm{H}$, Zheng $\mathrm{H}$, Wu J: Expression levels of microRNAs are not associated with their regulatory activities. Biol Direct 2011, 6:43. [http://dx.doi.org/10.1186/1745-6150-6-43]

45. Thomson JM, Newman M, Parker JS, Morin-Kensicki EM, Wright T, Hammond SM: Extensive post-transcriptional regulation of microRNAs and its implications for cancer. Genes Dev 2006, 20(16):2202-2207. [http://dx.doi.org/10.1101/gad.1444406]

46. Torres A, Torres K, Paszkowski T, Jodlowska-Jedrych B, Radomanski T, Ksiazek A, Maciejewski R: Major regulators of microRNAs biogenesis Dicer and Drosha are down-regulated in endometrial cancer. Tumour Biol 2011, 32(4):769-776. [http://dx.doi.org/10.1007/s13277-011-0179-0]

47. Dedes KJ, Natrajan R, Lambros MB, Geyer FC, Lopez-Garcia MA, Savage K Jones RL, Reis-Filho JS: Down-regulation of the miRNA master regulators Drosha and Dicer is associated with specific subgroups of breast cancer. Eur J Cancer 2011, 47:138-150. [http://dx.doi.org/10.1016/ j.ejca.2010.08.007]

48. Irizarry RA, Hobbs B, Collin F, Beazer-Barclay YD, Antonellis KJ, Scherf U, Speed TP: Exploration, normalization, and summaries of high density oligonucleotide array probe level data. Biostatistics 2003, 4(2):249-264. [http://dx.doi.org/10.1093/biostatistics/4.2.249]

49. Lockstone HE: Exon array data analysis using Affymetrix power tools and R statistical software. Brief Bioinform 2011, 12(6):634-644. [http:// dx.doi.org/10.1093/bib/bbq086]

50. Li H, Ruan J, Durbin R: Mapping short DNA sequencing reads and calling variants using mapping quality scores. Genome Res 2008 , 18(11):1851-1858. [http://dx.doi.org/10.1101/gr.078212.108]

51. R Development Core Team: R: A Language and Environment for Statistical Computing. Vienna, Austria: R Foundation for Statistical Computing; 2010. [http://www.R-project.org. [ISBN 3-900051-07-0].

52. Morgan M, Lawrence M, Anders S: ShortRead: Base classes and methods for high-throughput short-read sequencing data. [R package version 1.6.2].

53. Aboyoun P, Pages H, Lawrence M: GenomicRanges: Representation and manipulation of genomic intervals. [R package version 1.0.1].

54. Mortazavi A, Williams BA, McCue K, Schaeffer L, Wold B: Mapping and quantifying mammalian transcriptomes by RNA-Seq. Nat Methods 2008, 5(7):621-628. [http://dx.doi.org/10.1038/nmeth.1226]

55. Favero F: RmiR.Hs.miRNA: Various databases of microRNA Targets. [R package version 1.0.6]

56. Aulchenko YS, Ripke S, Isaacs A, van Duijn CM: GenABEL: an R library for genome-wide association analysis. Bioinformatics 2007, 23:1294-1296.

57. Aulchenko YS, Struchalin MV, van Duijn CM: ProbABEL package for genome-wide association analysis of imputed data. $B M C$ Bioinformatics 2010, 11:134.

58. Storey JD, Tibshirani R: Statistical significance for genomewide studies. Proc Natl Acad Sci U S A 2003, 100(16):9440-9445. [http://dx.doi. org/10.1073/pnas.1530509100]

doi:10.1186/1471-2164-13-383

Cite this article as: Geeleher et al:: The regulatory effect of miRNAs is a heritable genetic trait in humans. BMC Genomics 2012 13:383. 